\title{
Enfoque Epidemiológico sobre los Factores de Riesgo Biomecánico por Instalaciones Mecánicas en Edificios
}

\author{
EPIDEMIOLOGICAL APPROACH ABOUT BIOMECHANICAL RISK FACTORS FOR MECHANICAL INSTALLATIONS \\ IN BUILDINGS
}

Zorrilla Muñoz, Vanessa', Montero Puertas, Irene², Lorente Moreno, Rafael², Miranda García-Cuevas, Ma Teresa²

1. Ph.D in the Department of Mechanical, Energetic and Materials Engineering, Industrial Engineering School, University of Extremadura.

2. Professor Department of Mechanical, Energetic and Materials Engineering, Industrial Engineering School, University of Extremadura.

\section{RESUMEN}

El objetivo de este estudio es definir las causas biomecánicas de los trastornos músculo-esqueléticos de instalaciones mecánicas en edificios con el fin de conseguir objetivos como la validación y la información de precisión para el beneficio de las prevenciones de accidentes, y proporcionar información para los tratamientos de los trastornos músculo-esqueléticos.

Por estos medios, esta investigación analiza los trastornos, lesiones y daños músculo-esqueléticos aplicando métodos epidemiológicos descriptivos y analíticos. La estrategia consiste en los siguientes pasos: 1) Análisis de los accidentes de trabajo como método descriptivo. 2) Las preguntas de la entrevista marco como un método descriptivo, con el fin de obtener la recopilación de datos sobre los equipos de trabajo. 3) Informes en la literatura científica como método analítico con la influencias de la revisión de análisis clínico epidemiológico. 4) Estudio de caso-control, como método de análisis para los casos atípicos.

(Zorrilla V, Montero I, Lorente R, Miranda M, 2013. Enfoque Epidemiológico sobre los Factores de Riesgo Biomecánico por Instalaciones Mecánicas en Edificios. Cienc Trab. Ene-Abr; 15 [46]: 24-30).

Palabras claves: TRASTORNOS MÚSCULO-ESQUELÉTICOS, FACTORES DE RIESGO BIOMECÁNICO, EPIDEMIOLÓGICO.

\section{ABSTRACT}

The aim of this study is to define biomechanical causes of musculoskeletal disorders for mechanical installations in buildings in order to pursue goals such as the validation and precision information for the benefit in accident preventions, and provide information for the musculoskeletal disorders treatments. By these means, this research analyzes the musculoskeletal disorders, injuries, and damages by applying descriptive and analytical epidemiological methods. The strategy consists in the following steps: 1) Analysis of occupational accidents as descriptive method. 2) The framework interview questions as a descriptive method in order to obtain data collection about work teams. 3) Reports in the scientific literature as analytical method with the influences of epidemiological clinical analysis review 4) Case-control study as analytical method for the untypical cases.

Key words: MUSCULOSKELETAL DISORDERS, BIOMECHANICAL RISK FACTORS, EPIDEMIOLOGICAL

\section{INTRODUCCIÓN}

Los trastornos músculo-esqueléticos (TME's) abarcan una amplia gama de problemas de salud, siendo los de origen laboral un problema fundamental reconocido en el mundo. En general, los TME's ocupacionales constituyen una de las principales causas de lesiones y discapacidad en los países industrializados y en vías de desarrollo industrial ${ }^{1-4}$, figurando un buen ejemplo de las consecuencias que acarrea no tener en cuenta las exigencias de la ergonomía.
Correspondencia / Correspondence:

Vanessa Zorrilla Muñoz

Avda. Elvas s/n, 06071 Badajoz, España

Tel.: +34 685727950

e-mail: vzorrillam@gmail.com

Recibido: 24 Febrero 2013 / Aceptado: 03 de Marzo de 2013
El sector de instalaciones mecánicas en edificios parte del conocimiento de gran parte de los trabajos desarrollados en el sector de la construcción, ya que están profundamente vinculados a éste. Existen estudios sobre el sector de la construcción que indican un grave incremento de trastornos músculo-esqueléticos (TME's) asociados a los trabajos que se realizan. ${ }^{5}$ Las lesiones agudas osteomusculares en trabajos de la construcción dan lugar con frecuencia a sintomas crónicos y los efectos sustanciales que a largo plazo conllevan una reducción importante de la calidad de vida. ${ }^{6}$ Se ha demostrado en numerosos estudios que las causas que los producen están asociadas principalmente a los factores de riesgo biomecánico ${ }^{7,8}$, y la probabilidad se eleva al aumentar la causalidad al incorporarse los factores individuales ${ }^{9} \mathrm{y}$ genéticos, ambientales, organizativos y/o psicosociales $^{8,10}$, entre otros. Aun así, a pesar de los estudios realizados para el sector de la construcción, el nexo causa-etiología es una dimensión desconocida en las instalaciones mecánicas en edificios y se suelen atribuir en consideraciones causales y riesgos tipificados del sector de la construcción, que en realidad no han sido probados para los trabajos de instalaciones mecánicas de edificios. Por lo tanto, este estudio ha centrado su objetivo en elaborar un nexo causal directo de los (TME's) ocupacionales que afectan a los 
operarios de las instalaciones mecánicas de edificios, partiendo de la presunción de cada situación patogénica de TME que puede afectar a los operarios de las instalaciones mecánicas en edificios, es en realidad el resultado de una combinación de múltiples factores que dará lugar a la enfermedad y la posibilidad de que se materialice en el tiempo. ${ }^{5,11-13}$

\section{METODOLOGÍA}

La epidemiología es la disciplina que estudia la aparición de enfermedades en la población. Constituye una rama de las ciencias biomédicas que estudia las enfermedades y las causas por las cuales suceden las mismas. Siguiendo este contexto, la epidemiología ocupacional consiste en el estudio de la frecuencia de la enfermedad y su relación con las frecuencias de factores de riesgo, de carácter psicosocial u organizacional, físicos y biomecánicos, e individuales personales, centrados en aspectos relacionados con el puesto de trabajo, la organización, las actividades mecánicas o posturales, la edad, género, entre otras condiciones externas, junto a características específicas del individuo. ${ }^{14}$

Este estudio parte de la estrategia metodológica de dos ramas de la epidemiología, tal y como queda descrito en la Tabla 1: descriptiva $\mathrm{y}$ analítica.

El desarrollo se ha llevado a cabo mediante un muestreo de trabajadores del sector de instalaciones mecánicas en edificios que están expuestos a factores de riesgo biomecánicos ocupacionales.

El análisis epidemiológico descriptivo ha sido realizado recogiendo la información a tal efecto: Cuestionarios específicos e historia clínica. ${ }^{15}$ Para esto, en el proceso de la investigación se han planteado dos formas de aplicación de los métodos epidemiológicos: por una parte la elaboración de una entrevista cuestionario y, por otra, el estudio de accidentes de trabajo registrados en el grupo de operarios seleccionados.

Mediante la epidemiología analítica es posible establecer relaciones causales entre factores a los que se exponen personas y poblaciones y las enfermedades que presentan. Las medidas empleadas en este estudio de esta rama de la epidemiología son los factores de riesgo a que los operarios están expuestos, cuyo resultado es una razón de posibilidades. Es por ello que este estudio tiene en cuenta en el análisis epidemiológico analítico dos circunstancias. Por una parte, los casos que corresponden a la etiología investigada en las enfermedades, que proceden de estudios de poblaciones y las causas son detalladas para cada enfermedad, ya que la utilización de la etiología dentro de los sistemas de salud ocupacionales es una herramienta que sirve como base para el desarrollo de programas destinados al control de las enfermedades laborales en el propio lugar de trabajo. Por otra parte, se utiliza la epidemiología analítica de casos y controles para comprobar aquellos casos cuyo factor de exposición ha permitido la selección de un grupo de muestreo. La Tabla 1 a continuación mostrada, define resumidamente la estrategia de esta investigación.

Tabla 1.

Descripción de la estrategia metodológica para instalaciones mecánicas en edificios.

\begin{tabular}{ll} 
Métodos Epidemiológicos & \\
\hline Métodos Epidemiológicos & Investigación de accidentes \\
Descriptivos & Entrevista Estructurada \\
Métodos Epidemiológicos & Estudio de casos etiológicos definidos previamente \\
Analíticos & Estudio de casos y controles
\end{tabular}

\section{Investigación de accidentes}

En toda investigación de accidente se persigue conocer lo más fielmente posible que circunstancias y situaciones de riesgo de un entorno o puesto de trabajo que hayan posibilitado la materialización del accidente. El fin es la identificación de las causas a través del previo conocimiento de los hechos. Para ello, la investigación debe contemplar cinco etapas sucesivas: toma de datos, integración de los mismos, determinación de las causas, selección de las principales y ordenación de las mismas. ${ }^{16}$ Todo ello debe permitir eliminar las causas determinantes del accidente para evitar su repetición, así como permitirá aprovechar la experiencia adquirida para mejorar la prevención. El modelo que se ha seguido conduce a la secuencia lógica de la investigación de accidentes para recabar en las causas que ha producido el daño músculo-esquelético.

\section{La entrevista estructurada}

Una entrevista es un procedimiento diseñado para obtener información derivada de las respuestas verbales que una persona proporciona a preguntas orales ${ }^{17}$, con la finalidad de indagar sobre los hechos, conocimientos, opiniones, juicios y motivaciones. El tipo de entrevista utilizada ha constado de un cuestionario que ha servido de guía al entrevistador. Conjuntamente, se seleccionaron escalas adecuadas que siguieron los criterios de fiabilidad, validez y factibilidad. ${ }^{18}$ En el caso de la factibilidad, fue valorada mediante la consistencia interna según Alfa de Cronbach.

Las circunstancias que han llevado a utilizar una entrevista estructurada para la toma de datos han sido las siguientes ${ }^{19}:$ 1) La muestra ha estado constituida por personas que aunque puedan leer y escribir con facilidad, no entienden exactamente lo que se está preguntando, por lo que se requiere un entrevistador que aclare las cuestiones. 2) La entrevista vence los parámetros subjetivos, tratando de esclarecer lo más intimamente posible las preguntas. 3) Las personas entrevistadas no hacen las cosas de diferente manera en otras ocasiones; es por ello que se ha establecido previamente un periodo de observación y medición de las actividades. 4) No han existido barreras lingüisticas, puesto que los entrevistados considerados son todos de nacionalidad española. Además se ha utilizado un lenguaje verbal claro y sencillo. 5) Mediante la presentación del cuestionario a través de la entrevista se ha vencido el riesgo que existe en el empleo de material documentado sin considerar el sentido crítico.

Para realizar una valoración mediante el cuestionario, se ha recurrido al propósito de qué aspectos -que se pueden apreciar fácilmente como el dolor inicial y las puntuaciones de discapacidadpermiten confirmar el estado general de salud. ${ }^{20}$ Para esto se utilizaron cuestionarios específicos, partiendo de que la relación existente entre el TME y el dolor es la principal razón de la prolongación de los procesos de Incapacidad Temporal (IT) ${ }^{21}$, si bien es cierto que, según diversos autores, el dolor está mucho más afectado por las emociones que por la propia intensidad del dolor ${ }^{22,23}$, aspecto psicosocial que debería tenerse en cuenta en estudios psicosociales paralelos a este estudio. El modelo de cuestionario seleccionado ha partido de las cuestiones definidas en el "Cuestionario Nórdico" estandarizado. A diferencia de otros métodos, el "Cuestionario Nórdico" permite utilizar la entrevista en población activa cuando el dolor aún no supone una incapacidad $^{24}$, lo cual permite obtener información precisa de los TME's ocupacionales que pudieran llegar a aparecer en ciertas poblaciones. 


\section{Estudio de casos etiológicos definidos previamente}

El conocimiento de casos etiológicos dentro de los sistemas de salud que definen aproximaciones a teorías de causalidad, diagnóstico diferencial y toma de decisiones terapéuticas, o ensayos clínicos controlados resulta útil para conocer el desarrollo de los TME's que pudieran desarrollarse en análogas condiciones..$^{25}$ Bajo este marco, este estudio tiene en cuenta los casos definidos en investigaciones etiológicas previas, que han requerido la aplicación de la epidemiología para establecer los factores de riesgo biomecánicos y la intervención de otras ciencias sociales aplicadas para identificar los determinantes de los comportamientos organizativos e individuales que han dado lugar a la existencia de condiciones inseguras. No obstante, la etiología del desarrollo del TME laboral, debido al carácter multifactorial y su carácter acumulativo en el tiempo, añade dificultades para la predicción precisa, por lo que se requiere la diferenciación ${ }^{26}:$ 1) Se considera una enfermedad general cuando los factores individuales y extralaborales son la causa suficiente y necesaria para explicar el origen común de la enfermedad y no se presenta exposición a los factores de riesgo en el trabajo. 2) Pueden existir enfermedades generales relacionada con el trabajo, cuya etiología es fundamentalmente no ocupacional (individual o extralaboral) aunque los factores ocupacionales exacerben las manifestaciones de las enfermedades. 3) Las enfermedades profesionales están propiciadas por alguna de las siguientes situaciones: a) Las etiologías ocupacionales tiene mayor peso en la presentación de la enfermedad que los factores de origen común; b) La relación causal entre una exposición ocupacional y una enfermedad específica resulta clara (tanto médica como en general, legalmente); por lo tanto, se trata de una enfermedad profesional típica.

La dificultad principal ha concurrido en la determinación del nivel de predicción absoluto o multicausal de los factores de riesgo biomecánico que se relacionan con las enfermedades músculoesqueléticas laborales, de tal forma que sean una o múltiples causas las que lo producen. Con la especificación etiológica probada en ciencias médicas de la existencia de la enfermedad, es posible asimismo establecer una aproximación en la relación causal. Ahora bien, el principal compromiso inherente a toda investigación etiológica es el extremo de prudencia a la hora de emitir un juicio acerca de cualquier relación causal. Este campo multidisciplinar es lo que lleva a establecer que la causa -efecto de las patologías músculo-esqueléticas de origen laboral es más una tautología hasta el momento ${ }^{27}$ y no existan investigaciones epidemiológicas concisas para el sector de instalaciones mecánicas en edificios. Por el contrario, la apreciación del hecho multicausal favorece el abordaje de los problemas de salud desde diversas perspectivas, ya que al aceptarse que existen varias causas se puede actuar con diversas herramientas. En definitiva, tanto desde la acción preventiva como curativa.

Para poder concluir que un factor de riesgo contribuye al desarrollo de cierta enfermedad, es necesario que concurran a su vez ciertas circunstancias, que son: a) la asociación debe preceder al desenlace, es decir, que debe existir una secuencia temporal; b) la asociación no debe obedecer a alguna fuente de error sistémico (es decir, debe contener validez); la asociación entre exposición y desenlace debe ser estadísticamente significativa.$^{28}$ En definitiva, los factores de riesgo biomecánicos, aunque no lleguen a causar forzosamente enfermedades, sumados a otras múltiples circunstancias, aumentan el riesgo de producirla, de tal forma que cada factor contribuye un poco a la génesis de la enfermedad.

\section{Estudio de casos y controles}

Se ha aplicado la técnica de estudio de casos y controles a partir de la muestra de personas enfermas, parar llegar a estudiar los factores de exposición. De esta forma, se identifica a los enfermos (casos), que no tienen la enfermedad en estudio, pero que son similares a los casos, que se llamarán controles. La medida de asociación será el odds ratio $(\mathrm{OR}){ }^{30} \mathrm{El}$ estudio será calificado prospectivamente, ya que en este caso, a medida que se diagnostican, se incorporan al estudio. El estudio será llevado transversalmente, es decir, que los casos y controles se identificarán durante un lapso de tiempo dado antes de su inclusión en el estudio. Además, para que la comparación entre casos y controles se considere legitima en lo que concierne a la situación que presentan en términos de exposición, es necesario que todos los datos procedan de la misma población de referencia. ${ }^{29,30}$

La técnica que se ha seguido en el estudio de casos y controles es la siguiente:

1. Seleccionar una muestra de población con la enfermedad o con el problema de estudio. Estas enfermedades procederán del análisis realizado mediante los métodos epidemiológicos entre la población de estudio. A los individuos de esta muestra se les llama casos.

2. Seleccionar una muestra de la población de riesgo de enfermar, pero que esté libre de la enfermedad problema, que será el grupo control. El grupo de control es la muestra total objeto de estudio epidemiológico en esta investigación.

3. Medir las variables predictivas, que son los factores de riesgo. El cálculo se hará utilizando el programa Epidat 3.1, para el análisis de datos tabulados. Epidat es un programa de libre distribución desarrollado por el Servizo de Epidemioloxía de la Dirección Xeral de Innovación e Xestión da Saúde Pública de la Consellería de Sanidade (Xunta de Galicia) con el apoyo institucional de la Organización Panamericana de la Salud (OPS-OMS). ${ }^{31}$

\section{RESULTADOS}

Los trabajos estudiados del sector de instalaciones mecánicas en edificios han sido identificados en alguno de los siguientes grupos de trabajo:

a) Actividades relacionadas con el montaje de conductos, cuyo objetivo final es la construcción de redes de climatización completas, incluyendo el montaje de rejillas y/o toberas.

b) Actividades relacionadas con el montaje de maquinaria y tubería frigorífica, cuyo fin es la construcción de la instalación frigorífica, incluyendo aquellos equipos que conforman la instalación (evaporadoras y condensadoras).

c) Actividades relacionadas con el montaje de tubería de PCI, cuyo objetivo es la construcción de la instalación de PCI. Se excluyen aquellas operaciones que son desarrolladas mediante grúa de obra.

d) Actividades relacionadas con las instalaciones eléctricas, cuyo fin es la instalación de las redes eléctricas que se distribuyen a los sistemas de climatización. Se han excluido de este estudio las redes de suministro a máquinas y general del edificio, ya que estas forman parte de otros procesos eléctricos de la construcción del edificio.

e) Actividades relacionadas con la albañilería fina, cuyo objetivo es dar apoyo y rematar las instalaciones de climatización. 
Bajo el principio del análisis descriptivo obtenido a partir de estudios previos epidemiológicos y de laboratorio, se ha establecido una composición entre la enfermedad que puede llegar a desarrollarse ${ }^{26,32,33}$ y el factor o factores biomecánicos. ${ }^{34}$ Para los procesos estudiados en las instalaciones mecánicas en edificios, la descripción de las patologías en función de los factores de riesgo estudiados ha sido resumida como se describe en la Tabla 2, donde quedan descritos los factores biomecánicos que se definen a continuación: ${ }^{35}$

(1) Posturas forzadas y estáticas.

(2) Movimientos repetitivos.

(3) Levantamiento, manipulación manual y transporte de cargas.

(4) Movimientos manuales enérgicos de empuje y arrastre de cargas.

(5) Aplicación de fuerza y esfuerzo físico.

(6) Posturas estáticas puras.

Por otra parte, las lesiones músculo-esqueléticas que han sido relacionadas con estudios epidemiológicos previos, se refieren principalmente a ciatalgias, radiculopatía cervical, lumbalgia mecánica y contracturas musculares, las cuales han sido identificadas muy a menudo en relación a los factores de riesgo biomecánicos observados.

Tabla 2.

Matriz de relación entre factor biomecánico y enfermedad profesional.

\begin{tabular}{|c|c|c|c|c|c|c|}
\hline Lesiones & & & res & ecán & & \\
\hline músculo-esqueléticas & (1) & (2) & (3) & (4) & (5) & (6) \\
\hline Tendinitis (rotador y bicipital) & & $x$ & $x$ & $x$ & & \\
\hline Tendinitis (biceps) & & $\mathrm{x}$ & & & $\mathrm{x}$ & \\
\hline Epicondilitis & & $\mathrm{x}$ & & & & \\
\hline Epitrocleitis & & $\mathrm{x}$ & & & & \\
\hline Tendinitis tricipital & & $x$ & & & & \\
\hline Dedos resorte & & $x$ & & & & \\
\hline Otras tendinitis muñeca- & $x$ & $x$ & & & & $x$ \\
\hline Sindrome del Túnel Carpiano & $x$ & $x$ & & & & $x$ \\
\hline Sindrome del Canal Guyon & & $\mathrm{x}$ & & & & \\
\hline Sindrome del Canal Ulnar & & $x$ & & & & \\
\hline Sindrome del Pronador & & $x$ & & & & \\
\hline Sindrome del Opérculo & $x$ & $x$ & & & & $x$ \\
\hline Radiculopatía cervical & $x$ & $x$ & $x$ & $x$ & & $x$ \\
\hline Ciatalgia & $x$ & $x$ & $x$ & $x$ & $\mathrm{x}$ & $x$ \\
\hline Contractura muscular & $x$ & $x$ & $x$ & $x$ & $x$ & $x$ \\
\hline Dolor miofascial & $x$ & $x$ & & & & $x$ \\
\hline Miositis osificante & & $x$ & $x$ & $x$ & & \\
\hline Tensión muscular cervical & $x$ & $x$ & & & & \\
\hline Lumbalgia mecánica & $x$ & & $\mathrm{x}$ & $\mathrm{x}$ & $\mathrm{x}$ & $x$ \\
\hline Sindrome de Raynaud & & $\mathrm{x}$ & & & & \\
\hline Enfermedad art. degenerat. & & & & $x$ & & \\
\hline Bursitis subcutáneas & $x$ & & & & & $x$ \\
\hline Bursitis profunda & $x$ & $x$ & & & & $x$ \\
\hline Lesiones crónicas meniscos & & & $\mathrm{x}$ & $\mathrm{x}$ & & \\
\hline Ganglión carpiano & & $x$ & & & & \\
\hline Lesiones ligamentosas & $\mathrm{x}$ & $\mathrm{x}$ & & & & $\mathrm{x}$ \\
\hline
\end{tabular}

La muestra de la Tabla 3 indica el total de accidentes y enfermedades registradas, por una parte mediante la entrevista de trabajo $\mathrm{y}$, por otra, a través de la investigación de accidentes. El estudio de caso y control parte de la identificación concreta de lumbalgias, ya que por una parte, tres de los accidentes se corresponden al grupo de trabajo de instalación de maquinaria y tubería frigorífica que, a su vez, es el único grupo que realiza actividades que incluyen el factor de riesgo de movimientos manuales enérgicos (arrastre y empuje de cargas). Por otra parte, para el resto de casos
Tabla 3.

Lesiones músculo-esqueléticas registradas (para $n=34$ entrevistados y $n=6$ accidentes investigados).

\begin{tabular}{|c|c|c|c|c|c|c|}
\hline $\begin{array}{l}\text { Lesiones } \\
\text { músculo- } \\
\text { esqueléticas }\end{array}$ & $\begin{array}{l}\text { Montaje } \\
\text { de } \\
\text { conductos }\end{array}$ & $\begin{array}{l}\text { Montaje } \\
\text { de tuberia } \\
\text { de PCl }\end{array}$ & $\begin{array}{l}\text { Montaje de } \\
\text { maquinaria } \\
\text { y tuberia }\end{array}$ & \begin{tabular}{l}
\multicolumn{1}{c}{ Casos } \\
Instalaciones \\
eléctricas
\end{tabular} & $\begin{array}{l}\text { Albañilería } \\
\text { fina }\end{array}$ & $\begin{array}{l}\text { Número } \\
\text { de } \\
\text { operarios }\end{array}$ \\
\hline Lumbalgias & 1 & 1 & 3 & & 1 & 6 \\
\hline 乞 Tendinitis & 1 & & & 1 & 1 & 3 \\
\hline 凹్ Epicondilitis & 2 & & & & & 2 \\
\hline হa Ciatalgia & 1 & & & & & 1 \\
\hline 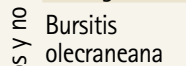 & 1 & & & & & 1 \\
\hline $\begin{array}{l}\text { पू Contractura } \\
\frac{\tilde{\partial}}{x} \text { brazo-hombro }\end{array}$ & & & & & & 1 \\
\hline $\begin{array}{l}\text { लै Contractura } \\
\text { cuello }\end{array}$ & & 1 & & 1 & & 1 \\
\hline
\end{tabular}

no se ha obtenido ningún caso de exposición que pueda llegar a relacionarse con un factor en concreto. Por último, todos los grupos estudiados llevan asociados los factores de riesgo biomecánicos identificados en la Tabla 2, excepto el caso de montaje de maquinaria y tubería frigorífica, donde existe un factor de riesgo que sólo en este grupo es experimentado, lo cual ha permitido seleccionar una muestra de población expuesta y no expuesta. Mediante el estudio de caso y control, se ha analizado la relación entre dicho factor y la lumbalgia mecánica.

En la Tabla 4 se ha representado la matriz sobre el estudio de casos y controles para el trastorno lumbalgias. Para la muestra a comparar, los datos fueron recopilados de la siguiente manera:

- Se registraron 3 lumbalgias en el grupo de trabajo de montaje y maquinaria frigorífica que realiza una actividad excepcionalmente, que consiste en arrastrar y empujar condensadoras para ubicarlas en la posición adecuada a los planos de construcción. Esta situación se lleva a cabo porque la grúa no puede instalar las condensadoras en el lugar apropiado sin la ayuda mecánica humana.

- De las 5 personas identificadas en la actividad de arrastre y empuje de condensadoras, se relacionó un trabajador que había indicado que había padecido lumbalgia. Los otros dos -que también pertenecian al grupo de montaje de maquinaria y tuberia-, en el momento del registro de accidentes no se encontraban entre el grupo muestreado en la actividad. Sin embargo, al preguntarles en la investigación del accidente, uno de ellos indicó que sí había realizado este tipo de actividad y el otro contestó que no. Con esto, fueron registrados 2 casos de lumbalgias y la muestra de 5 operarios expuestos es ampliada a un total de 6 .

- Para disponer de un grupo de controles, se partió de la muestra de 150 personas registradas durante la observación de las actividades.

Los resultados fueron obtenidos a través del programa Epidat 3.1, que presenta las siguientes medidas de frecuencia para una tabla de contingencia de tipo $2 \times 2$ en un estudio de casos y controles:

- Proporción de casos expuestos; en este caso, los afectados por lumbalgia que han estado expuestos al factor de empuje y arrastre de condensadoras y los que no han realizado esta actividad; por lo tanto, no han estado expuestos al factor de exposición en relación al arrastre y empuje de condensadoras.

- Proporción de controles expuestos, es decir, la muestra de personas expuestas al factor de exposición (arrastre y empuje 
Tabla 4.

Resultados y matriz de estudio de casos y controles mediante Epidat 3.1.

\begin{tabular}{|c|c|c|c|c|}
\hline & & $\begin{array}{l}\text { Casos con } \\
\text { lumbalgias }\end{array}$ & $\begin{array}{l}\text { Controles sin } \\
\text { lumbalgias }\end{array}$ & Totales \\
\hline \multicolumn{2}{|c|}{$\begin{array}{l}\text { Expuestos a movimientos } \\
\text { manuales enérgicos }\end{array}$} & 2 & 4 & 6 \\
\hline \multicolumn{2}{|c|}{$\begin{array}{l}\text { No expuestos a movimientos } \\
\text { manuales enérgicos }\end{array}$} & 4 & 140 & 144 \\
\hline \multirow[t]{2}{*}{ Totales } & \multicolumn{2}{|r|}{6} & 144 & 150 \\
\hline & Estimación & IC $95 \%$ & & \\
\hline $\begin{array}{l}\text { Proporción de } \\
\text { casos expuestos }\end{array}$ & & --- & --- & \\
\hline $\begin{array}{l}\text { Proporción de } \\
\text { controles expuestos }\end{array}$ & 0,027778 & --- & --- & \\
\hline \multirow[t]{3}{*}{ Odds ratio } & \multirow[t]{2}{*}{17,500000} & 2,447946 & 125,104867 & (Wolff) \\
\hline & & 2,942219 & 111,831798 & (Cornfield) \\
\hline & \multicolumn{2}{|r|}{ Estimación } & IC 95\% & \\
\hline \multirow{2}{*}{\multicolumn{2}{|c|}{$\begin{array}{l}\text { Fracción atribuible en expuestos } \\
\text { Fracción atribuible poblacional }\end{array}$}} & 0,942857 & 0,591494 & 0,992007 \\
\hline & & 0,314286 & $-0,208249$ & 0,610838 \\
\hline \multicolumn{2}{|c|}{ Prueba Ji-cuadrado de asociación } & Estadístico & Valor $\mathrm{P}$ & \\
\hline \multirow{2}{*}{\multicolumn{2}{|c|}{$\begin{array}{l}\text { Sin corrección } \\
\text { Corrección de Yates }\end{array}$}} & 14,0046 & \multirow{2}{*}{$\begin{array}{l}0,0002 \\
0,0074\end{array}$} & \\
\hline & & 7,1777 & & \\
\hline \multicolumn{2}{|c|}{ Prueba exacta de Fisher } & Valor P & & \\
\hline \multirow{2}{*}{\multicolumn{2}{|c|}{$\begin{array}{l}\text { Unilateral } \\
\text { Bilateral }\end{array}$}} & 0,0187 & & \\
\hline & & 0,0187 & & \\
\hline
\end{tabular}

de condensadoras) que no han resultado afectadas y la muestra de personas que no están expuestas al factor y no han padecido lumbalgias.

Las medidas de asociación que se presentan en los resultados son:

- Odds ratio e intervalo de confianza para una incidencia acumulada. El odds ratio en el programa se utiliza para un intervalo de confianza aproximado por el método de Woolf y para un intervalo de confianza aproximado por el método de Cornfield.

El intervalo de confianza (IC) será de un 95\%.

- Fracción atribuible o prevenible para la población expuesta e intervalo de confianza.

En cuanto a las medidas de impacto para la asociación entre variables:

- Fracción atribuible o prevenible para la población.

Y por último, las medidas de significación estadística:

- Prueba Ji-cuadrado de asociación, con y sin corrección.

- Prueba exacta de Fisher y valor de p, unilateral y bilateral.

Asimismo, la interpretación de este resultado en términos epidemiológicos indica que existe una asociación entre las actividades donde se realizan movimientos enérgicos (arrastre y empuje) y la lumbalgia, donde el riesgo de desarrollar este trastorno en trabajadores del grupo montaje de maquinaria y tubería frigorífica (expuestos) es 17,5 veces más elevado que el resto de trabajadores (no expuestos) para un intervalo de confianza (IC) del 95\%. Es importante tener en cuenta que en los resultados los intervalos de confianza de Woolf y Cornfield están en ambos extremos por debajo de 1 .

Las fracciones previstas en expuestos y en la población tienen la misma interpretación que la fracción atribuible, en tanto que las pruebas estadísticas con valores de $\mathrm{p}$ pequeños (en particular, menores a 0,05) indican que se puede descartar el azar como explicación de la asociación observada con una reducida probabilidad de cometer el error de primer tipo (hacer un rechazo inde- bido). Otro argumento en contra de las asociaciones del azar se enfoca en que la mayoría de los resultados tienen patrones coherentes y que los factores analizados han sido identificados como asociados con los trastornos músculo-esqueléticos en estudios anteriores. $^{36}$

\section{CONCLUSIONES}

Una de las funciones generales de la vigilancia de la salud en el trabajo es la identificación de casos individuales de enfermedades y lesiones profesionales, para así estar en condiciones de detectar y evaluar a otras personas dentro del mismo lugar de trabajo que puedan estar en situación de riesgo. Este proceso permite asimismo instaurar medidas de control para reducir las condiciones peligrosas asociadas con la aparición del caso índice -es decir, la primera persona enferma o lesionada en un lugar de trabajo que recibe asistencia médica y, por consiguiente, la primera en llamar la atención sobre la existencia de un riesgo en el lugar de trabajo y sobre otros trabajadores que puedan encontrarse también en situación de riesgo-. Otro objetivo de la identificación de casos es conseguir que la persona afectada reciba un seguimiento clínico adecuado. ${ }^{37,38}$

En la investigación de accidentes y enfermedades se ha comprobado la relación causal que, además, confirma la relación de enfermedades relacionadas en estudios etiológicos previos. Es interesante comprobar que los resultados obtenidos en la entrevista, así como en los accidentes, apuntan hacia la región dorsolumbar y región de cuello-hombros como zonas más afectadas. Esto que a priori no parece importante, sí lo es en realidad, ya que la reiteración de los casos es lo que supone a largo plazo la cronicidad. ${ }^{12}$ Por otra parte, también se ha justificado el porqué de estos procesos dolorosos y el factor biomecánico asociado al proceso, mediante la discusión entre actividad y posible lesión, que además ha permitido relacionar los resultados obtenidos mediante la entrevista y la investigación de accidentes. Cabe indicar que estos datos concuerdan con los obtenidos por sectores de la VI Encuesta Nacional del 2006, que reflejaban que la región lumbar es la zona más frecuentemente afectada por los TME's en el sector. ${ }^{39}$

Los estudios de caso y control son muy útiles para relacionar el factor de exposición con un grupo de personas. En el caso de esta investigación, el único factor de exposición posible para llevar a cabo el estudio comparativo de casos y controles para el TME de lumbalgias ha sido la actividad relacionada con el arrastre de cargas, donde se ha querido evidenciar si existe o no una justificación trazable con el grupo de montaje de maquinaria y tubería frigorífica. El resultado es que la probabilidad de exposición es muy superior en este grupo que en el grupo de trabajadores no expuestos, aunque no se puede descartar el azar. Tal y como se ha venido justificando hasta ahora, los factores de tipo biomecánico determinan la posibilidad de que las enfermedades profesionales se produzcan; esto, junto con otros factores que pudieran añadirse, es lo que hace que acontezca el trastorno músculo-esquelético. La realidad es que en el sector de instalaciones mecánicas en edificios los TME's se desarrollarán debido a la existencia de los factores biomecánicos y otros factores añadidos que han sido mencionados previamente en este estudio, como los psicosociales, organizativos, genéticos, individuales y ambientales, entre otros. Otro aspecto importante a tener en cuenta es que resulta evidente que la intervención de los factores dentro de la organización del trabajo contri- 
buye al desarrollo de TME's de origen laboral, no solamente en el trabajador (por ejemplo, formación/endurecimiento de trabajo). También se suscita el hecho de que el apoyo y participación activa de las personas en situación de riesgo y otras partes interesadas en las organizaciones influencia en buena medida el desarrollo de patologías. ${ }^{40}$ La propia organización del trabajo en la empresa contribuirá en mayor o menor medida a que los factores biomecánicos sean más o menos partícipes en el desarrollo y aparición de lesiones. ${ }^{41}$ Las empresas constituyen una excelente fuente de información sobre las exposiciones profesionales y la aparición de enfermedades relacionadas con el trabajo. Sin embargo, muchas de estas empresas carecen de los conocimientos especializados necesarios para evaluar el nivel de exposición en el lugar de trabajo o para determinar si una enfermedad está relacionada con el trabajo. Además, los desincentivos económicos ligados al hallazgo del origen profesional de una enfermedad pueden desalentar a las organizaciones a hacer un uso adecuado de esa información. El posible conflicto de intereses entre la salud financiera de la empresa y la salud física y mental de los trabajadores representa un importante obstáculo para la mejora de la vigilancia de las enfermedades profesionales. ${ }^{42}$ Por otra parte, lograr una reducción significativa y sostenida requiere esfuerzos concentrados en la jerarquía de influencias de causas y factores de riesgo, en los que el sector de la construcción muestra muchas carencias. ${ }^{43}$

Por último, la metodología planteada en este estudio pudiera resultar de gran utilidad para expertos y técnicos de prevención cualificados en las empresas ya que conlleva un gasto mínimo y los resultados que se obtienen del estudio epidemiológico pueden llegar a ser muy favorables. Sería interesante, en futuros estudios, continuar con los casos epidemiológicos de estudios de casos y controles o incluso plantear estudios de cohortes que permitan reunir más datos para la búsqueda de factores causales asociados a determinados grupos de trabajo muy específicos en este sector $u$ otros paralelos. La dinámica vendría dada mediante la recopilación de datos en el proceso de asistencia sanitaria durante un período de estudio más amplio. También resultaría de gran valor para estudios posteriores si las investigaciones de accidentes y enfermedades o reportes de TME's se observan y analizan minuciosamente con el fin de recabar en datos de sectores específicos y desarrollo de actividades en lugar del establecimiento de clasificaciones tipo para sectores con características comunes, pero en las que no se ha llegado a identificar notoriamente los trabajos desarrollados.
1. Genaidy $A M$, al-Shedi $A A$, Shell RL. Ergonomic risk assessment: preliminary guidelines for analysis of repetition, force and posture. J Hum Ergol. 1993;22(1):45-55.

2. Kemmlert K. Labor Inspectorate Investigation for the Prevention of Occupational Musculo-Skeletal Injuries (Dissertation). Solna,Sweden: National Institute of Occupational Health;1994.

3. Iran. National Research Center of Medical Sciences of Iran. NRCMSI. National Health Survey of Iran: Overall Country NHSI. Tehran: NRCMSI; 2001.

4. Shahnavaz H. Workplace injuries in the developing countries. Ergonomics. 1987;30(2):397-404.

5. Agencia Europea para la Seguridad y la Salud en el Trabajo; FACTS 71: Introducción a los Trastornos musculoesqueléticos de origen laboral [on line]. Bilbao: OSHA; 2007 [consultado marzo 2013]. (FACTS 71; 2). Disponible en: https://osha.europa.eu/es/publications/factsheets/71

6. Punnett L, Wegman DH. Work-related musculoskeletal disorders: the epidemiologic evidence and the debate. J Electromyogr Kines. 2004;14(1):13-23.

7. España. Instituto de Trabajo y Seguridad Social. ITSS. Guia de Actuación Inspectora en Factores Ergonómicos. En: Guía de Actuación Inspectora en Factores Ergonómicos. Madrid: ITSS; 2006.

8. Mondelo PR, Bombardo P, Blasco EG. Ergonomía 3. Diseño de puestos de trabajo. Barcelona: Universitat Politècnica de Catalunya; 1999.

9. Cole DC, Rivilis I. Individual factors and musculoskeletal disorders: a framework for their consideration. J Electromyogr Kines. 2004;14(1):121-127.

10. Nancy NM. Psychosocial Factors in Musculoskeletal Disorders. Crit Care Nurs Clin North Am. 200719(2):145-153.

11. Buckle PW, Devereux JJ. The nature of work-related neck and upper limb musculoskeletal disorders. Appl Ergon. 2002;33(3):207-217.

12. Bugarin $R$, Galego $P$, Garcia $A$, Rivas $P$. Musculoskeletal disorders in dental professionals. RCOE. 2005;10(5-6):561-566.
13. Hagberg M, Silverstein BA, Wells RV, Smith MJ, Hendrick HW, Carayon P et al. Work Related Musculoskeletal Disorders. London: Taylor \& Francis;1995.

14. Ahlbom A, Alfredsson L, Alfvén T, Bennet A. Fundamentos de epidemiologia. Madrid: Siglo XXl; 2007.

15. Rayo MA, Damián J. Método Epidemiológico. Madrid: Instituto de Salud Carlos III-E.N.S.; 2009.

16. Bestraten M, Gil A, Piqué T. NTP 592: La gestión integral de los accidentes de trabajo (I): tratamiento documental e investigación de accidentes. Notas Técnicas de Prevención. Madrid: INSHT; 1999. p.1-8.

17. Dessler G. Administración de personal. Naucalpan de Juárez: Pearson Educación; 2001.

18. Saunders $M$, Lewis $P$, Thornhill $A$. Research methods for bussiness students. Harlow: Pearson; 2009.

19. Urbano C, Yuni J. Técnicas para investigar 2. Córdoba: Brujas; 2006.

20. Jezukaitis $P$, Kapur D. Management of occupation-related musculoskeletal disorders. Best Pract Res Cl Rh. 2011; 25(1):117-129.

21. Araña SM, Patten S. Trastornos Músculo-Esqueléticos, Psicopatología y Dolor. En: Trastornos Músculo-esqueléticos, psicopatología y dolor. Madrid: Secretaría del Estado para la Seguridad Social-Ministerio de Trabajo e Inmigración; 2011. pp. 1-13

22. Barber J. Tratamiento del dolor mediante hipnosis y sugestión: una guia clínica. Bilbao: Desclée de Brouwer; 2000.

23. Patterson DR. Clinical Hypnosis for Pain Control. Washington DC: American Psychological Association; 2010.

24. Kuorinka $I$, Jonsson $B$, Kilbom $A$, Vinterberg $H$, Biering-Sorensen $F$, Andersson $G$ et al. Standardised Nordic questionnaires for the analysis of musculoskeletal symptoms. Appl Ergon.1987;18(3):233-237.

25. Kazem SZ. Fundamentals of clinical methodology: 2. Etiology. Artif Intell Med.1998;12(3):227-270. 


\section{REFERENCIAS}

26. Arbeláez JA, Sierra MI. Enfermedades Musculoesqueléticas en el Trabajo. En: Rehabilitación en Salud. 2a ed. Medellín: Universidad de Antioquia; 2008. pp. 837-856.

27. Benavides $F C$, Garcia AM, Ruiz-Frutos, $C$. La salud y sus determinantes. En: Benavides F, García A, Ruiz-Frutos C, Delclós J. editores. Salud Laboral. Conceptos y técnicas para la prevención de riesgos laborales. $3^{a}$ ed. Barcelona: Elsevier Masson; 2007. pp. 3-14

28. Arcos P. Metodología de la investigación. En: Arcos P.; Castro R. editores. Manual de Medicina de Urgencia y Emergencia. Vol. 2. Oviedo: Universidad de Oviedo; 2009. pp. 181-196

29 Rothman KJ. Modern Epidemiology. Boston: Little, Brown and Company; 1986.

30. Szklo M, Nieto FJ. Epidemiology: Beyond the Basics. 2nd ed. London: Jones and Bartlett Publishers; 2007.

31. España. Xunta de Galizia. EPIDAT: Análise Epidemiolóxica de Datos[online]. Santiago de Compostela: Servizo Galego de Saúde-OPS; 2011. [Consultado 19 ene 2012]. Disponible en: http://www.sergas.es/MostrarContidos_N3_ T01.aspx?ldPaxina $=62713$

32. Buckle P, Deveroux J. Work-related neck and upper limb musculoskeletal disorders. Luxembourg: European Agency for Safety and Health at Work; 1999.

33. Serranheira F. Lesões Músculo-Esqueléticas Ligadas ao Trabalho:que métodos de avaliação do risco?. [Tese Ph.D.]. Lisboa: Universidade Nova de Lisboa, Escola Nacional de Saúde Pública; 2007.

34. Warren N. Work stress and musculoskeletal disorder etiology: The relative roles of psychosocial and physical risk factors. Work. 2001;17(3):221-234.

35. Instituto Nacional de Seguridad e Higiene del Trabajo. INSHT. Portal de los Trastornos musculoesqueléticos [on line]. Madrid: INSHT; 2011. [Consultado 19 Sep 2012]. Disponible en: http://www.insht.es/portal/site/Musculo Esqueleticos/.
36. Fredriksson K, Bildt C, HÃ€gg G, Kilbom Ã. The impact on musculoskeletal disorders of changing physical and psychosocial work environment conditions in the automobile industry. Int J Ind Ergon. 2001;28(1):31-45.

37. Baker EL, Melius JM, Millar JD. Surveillance in Occupational Illness and Injury: Concepts and Content. Am J Pub Health.1989;79(Supplement):9-11.

38 Castorina JS, Rosenstock LR. Physician Shortage in Occupational and Environmental Medicine. Ann Int Med.1990;113(12):983-986.

39. European Agency for Safety and Health at Work. EASHW. OSH in figures: Work-related musculoskeletal disorders in the EU - Facts and figures. Luxembourg: Publications Office of the European Union; 2010.

40. National Research Council. NRC. Work-related musculoskeletal disorders: report, workshop summary and workshop papers. Washington DC: National Academy Press; 1999.

41. Díaz P. Prevención de Riesgos Laborales; Seguridad y salud laboral. Paracuellos del Jarama: Paraninfo. 2009.

42. Markowitz SB. Sistemas de vigilancia y notificación de enfermedades profesionales. En: Enciclopedia de Salud y Seguridad en el Trabajo. Vol. 1. Madrid: OIT; 2001. pp. 32.32-32.38.

43. Haslam RA, Hide SA, Gibb AGF, Gyi DE, Pavitt $T$, Atkinson $S$ et al. Contributing factors in construction accidents. Appl Ergon. 2005;36 (4):401-415

\section{Referencias complementarias:}

1. European Foundation for the Improvement of Living and Working Conditions. EFILWC. Fourth European Working Conditions Survey. Dublin: Office for Official Publications of the European Communities; 2007.

2. Rothman KJ. Greenland S, Lash TL. Case-Control Studies. In: Encyclopedia of Quantitative Risk Analysis and Assessment. New Jersey: John Wiley \& Sons; 2008. 